\section{THE CHANGING FACE OF ANTI-PLATELET PRESCRIPTIONS IN ENGLAND: 1998-2015}

${ }^{1}$ Christopher P Primus*, ${ }^{1}$ Krishnaraj S Rathod, ${ }^{2}$ Hitesh C Patel, ${ }^{3}$ Ketna Patel, ${ }^{3}$ Sanjeev Bhattacharyya, ${ }^{4}$ Carl Hayward. ${ }^{1}$ Barts Heart Centre and Queen Mary, University of London; ${ }^{2}$ Royal Brompton NHS Trust; ${ }^{3}$ Barts Heart Centre; ${ }^{4}$ Barts Heart Centre and Royal London Hospital

\subsection{6/heartjnl-2017-311726.98}

Introduction Aspirin has been widely prescribed for primary and secondary prevention of cardiovascular (CV) events for decades. In recent years, attitudes around its efficacy and safety have changed in two important areas: primary prevention of vascular events and stroke prevention in patients with atrial fibrillation (AF). In particular, two meta-analyses and a large randomised-control trial (RCT) suggested that in primary prevention, the modest reduction in $\mathrm{CV}$ events afforded by aspirin was accompanied by a modest increase in the risk of serious bleeding

Aim We sought to describe the trends of aspirin and other anti-platelet prescribing in England over the 18 year period, 1998-2015.

Methods We conducted a comprehensive nationwide retrospective study. Data were obtained from the Prescription Cost Analysis system, which holds information on every prescription dispensed in the community in England, covering a population of more than 50 million $(\mathrm{m})$ people. We obtained data for 5 anti-platelet agents from 1998-2015.

Results From 1998-2008 annual aspirin prescriptions increased linearly, peaking at 33.9 million in 2009 (Figure 1). Since 2009 , aspirin prescribing has fallen to $28 \mathrm{~m}$ (2015). Over this latter period, clopidogrel use has risen to a peak of $8.2 \mathrm{~m}$ scripts in 2015. More recently prasugrel and ticagrelor have been prescribed but comprise a small proportion of total prescriptions (Table 1). Total anti-platelet prescriptions have plateaued since 2009 (39.3m in 2009 and $37.4 \mathrm{~m}$ in 2015).
Conclusion Anti-platelet prescribing increased linearly from 1998-2009 and plateaued thereafter. The decline in aspirin prescribing coincides with the publication of two meta-analyses and a large RCT that cast doubt on its efficacy in primary prevention and raised concerns about the increased risk of bleeding in this population (Antithrombotic Trialists Collaboration 2009; de Berardis et al 2009; Fowkes et al 2010). Our data are limited given the indication for each prescription is unavailable, but we can hypothesise that the reduction in aspirin use from 2009 is due to a reduction in primary prevention prescribing.

Changes in CV guidelines have had further impact on total anti-platelet prescribing. NICE guidance in AF advocates formal anticoagulation for stroke prevention, with no role for anti-platelets (NICE CG180, 2014). In the absence of AF, clopidogrel now supercedes aspirin \pm dipyridamole in the prevention of occlusive events in stroke and peripheral arterial disease (NICE TA210, 2010). With rising rates of coronary intervention, these changes explain escalating clopidogrel use over this period.

Whilst the new anti-platelet agents contribute relatively little to current prescribing, the landscape is likely to change as their role beyond acute myocardial infarction is investigated.

\section{POWERFORM FOR REAL-TIME ENTRY OF ROUTINE CARDIAC OUTPATIENT DATA INTO THE ELECTRONIC HEALTH RECORD: APPLICATION FOR AUDIT AND RESEARCH}

${ }^{1}$ Neil Bodagh*, ${ }^{2}$ Andrew Archbold, ${ }^{1}$ Roshan Weerackody, ${ }^{3}$ Mike Barnes, ${ }^{3}$ John Robson, ${ }^{2}$ Adam Timmis. ${ }^{1}$ Bart's Heart Centre; ${ }^{2}$ Bart's Heart centre; ${ }^{3}$ Queen Mary University

10.1136/heartjnl-2017-311726.99

Abstract 99 Table 1 Prescriptions of each anti-platelet agent as a proportion of total anti-platelets prescribed

\begin{tabular}{|l|c|c|c|c|c|c|c|c|c|c|c|}
\hline & $\mathbf{2 0 0 5}$ & $\mathbf{2 0 0 6}$ & $\mathbf{2 0 0 7}$ & $\mathbf{2 0 0 8}$ & $\mathbf{2 0 0 9}$ & $\mathbf{2 0 1 0}$ & $\mathbf{2 0 1 1}$ & $\mathbf{2 0 1 2}$ & $\mathbf{2 0 1 3}$ & $\mathbf{2 0 1 4}$ & $\mathbf{2 0 1 5}$ \\
\hline Aspirin & 86.6 & 86.2 & 86.3 & 86.5 & $\mathbf{8 6 . 2}$ & $\mathbf{8 5 . 5}$ & 84.2 & 82.0 & 79.7 & 77.4 & 75.0 \\
\hline Clopidogrel & 10.1 & 10.4 & 10.0 & 9.4 & 9.4 & 9.6 & 11.0 & 13.9 & 16.8 & 19.4 & 22.0 \\
\hline Dipyridamole & 3.3 & 3.4 & 3.7 & 4.0 & 4.4 & 4.9 & 4.6 & 3.7 & 2.8 & 2.2 & 1.8 \\
\hline Ticagrelor & 0.0 & 0.0 & 0.0 & 0.0 & 0.0 & 0.0 & 0.0 & 0.1 & 0.4 & 0.7 & 1.0 \\
\hline Prasugrel & 0.0 & 0.0 & 0.0 & 0.0 & 0.0 & 0.0 & 0.2 & 0.3 & 0.3 & 0.2 & 0.2 \\
\hline
\end{tabular}

Total number of anti-platelet prescriptions in primary care in England 1998-2015

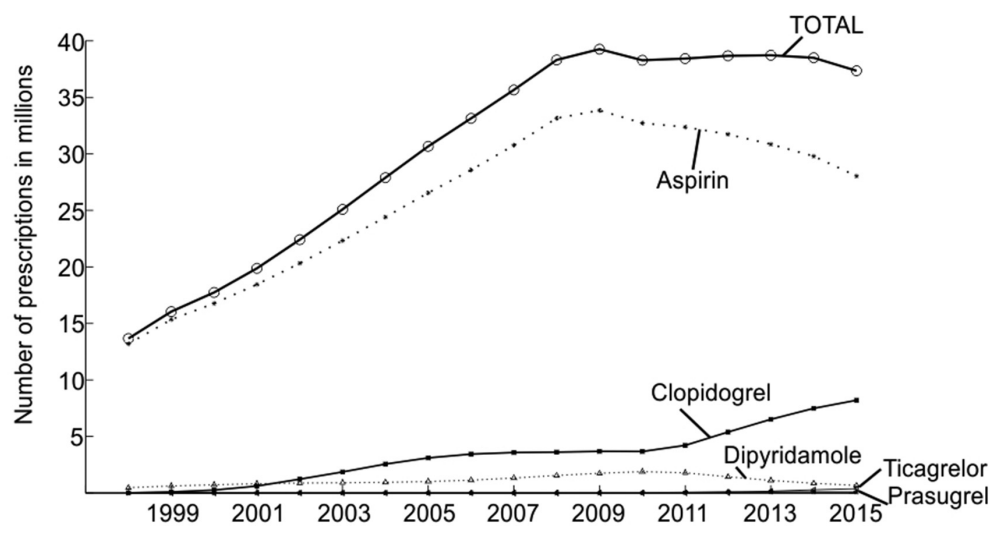

Abstract 99 Figure 1 
Background The electronic health record (EHR) is the major repository of clinical data in the NHS. It is a huge potential resource but remains severely under-utilised to the point that almost none of the UK's audit and research output is based on routinely collected clinical data. The reasons are complex but ultimately reflect the fact that these data are rarely entered into the EHR in a form that allows for their organised storage and digital download.

Methods We have developed a SNOMED-based electronic powerform comprising a user-friendly interface for real-time entry of clinical data into the hospital EHR during cardiac outpatient consultation. Our aim was to capture outpatient clinical data in a form that allows for automatic development of summary patient reports and for batch download of deidentified data for audit and research.

Results During the first 4 months after installation of the powerform, consultant utilisation averaged $60 \%$ for the 327 new patients seen during that period. Presenting symptoms, examination findings, investigations, diagnosis, initial treatment and disposal (>120 fields) were entered in real time during consultation and a structured summary report was developed. This was made available for electronic transfer directly into the patients EMIS file in the primary care record, permitting same-day delivery of the report and obviating the need for a dictated clinic letter. Batched download of the digital data was successful, with sample analytic findings as follows:

Patient ethnicity South Asian 44\%, white 34\%, black 15\% Presenting symptom Chest pain 41\%, dyspnoea 11\%, palpitations 10\%, dizzy attacks/syncope 8\%, hypertension 7\%

Diagnosis Non-cardiac chest pain 24\%, angina 11\%, coronary disease $7 \%$

Disposal discharged to GP 74\%, follow-up appointment $11 \%$, cath lab waiting list 6\%, referral to specialist clinic $9 \%$.

Ongoing are surveys of satisfaction with the power forms (cardiologists) and with the summary reports (GPs).
Conclusion This is the first report of powerform development for entry of routinely collected cardiac outpatient data into the hospital EHR. The data are stored in a form that permits: (1) automatic generation of a summary report for same-day delivery into the primary care record and (2) batch download of de-identified digital data for audit. Integration of the system with programmes of generic patient consent will open up the EHR to real-world clinical research.

\section{EFFECT OF OXYGEN THERAPY IN EARLY NEOTNATAL LIFE ON CAPILLARY MICROCIRCULATION AND BLOOD PRESSURE IN LOW BIRTH WEIGHT INFANTS}

${ }^{1}$ Rajendra Raghuraman, ${ }^{2}$ Donovan Duffy, ${ }^{2}$ saac Manyondaa, ${ }^{1}$ Tarek Antonios*. ${ }^{1} S t$ George's, University of London; '2St George's University Hospitals NHS Foundation Trust

\subsection{6/heartjnl-2017-311726.100}

Background Low birth weight (LBW) is a risk factor for adult essential hypertension, diabetes mellitus, obesity and cardiovascular disease mortality in later life. Individuals with history of LBW have many abnormalities in their blood vessels including a reduction in microvascular density or rarefaction. Capillary rarefaction (CR) is a hallmark of essential hypertension and evidence suggests that $\mathrm{CR}$ is a primary structural abnormality that precedes the onset of the rise in blood pressure (BP) as it is found in normotensive individuals at high risk of developing hypertension. We recently found that LBW infants have higher and not lower capillary density at birth. We hypothesised that LBW infants will undergo significant CR in early life triggered or exaggerated by oxygen therapy.

Methods We studied 26 LBW infants and 14 normal birth weight infants (NBW) as controls. Of the LBW infants, only 10 received oxygen therapy. We used a handheld video capillaroscopy system (HVCS) to measure functional or basal (BCD) and structural or maximal capillary density (MCD) on

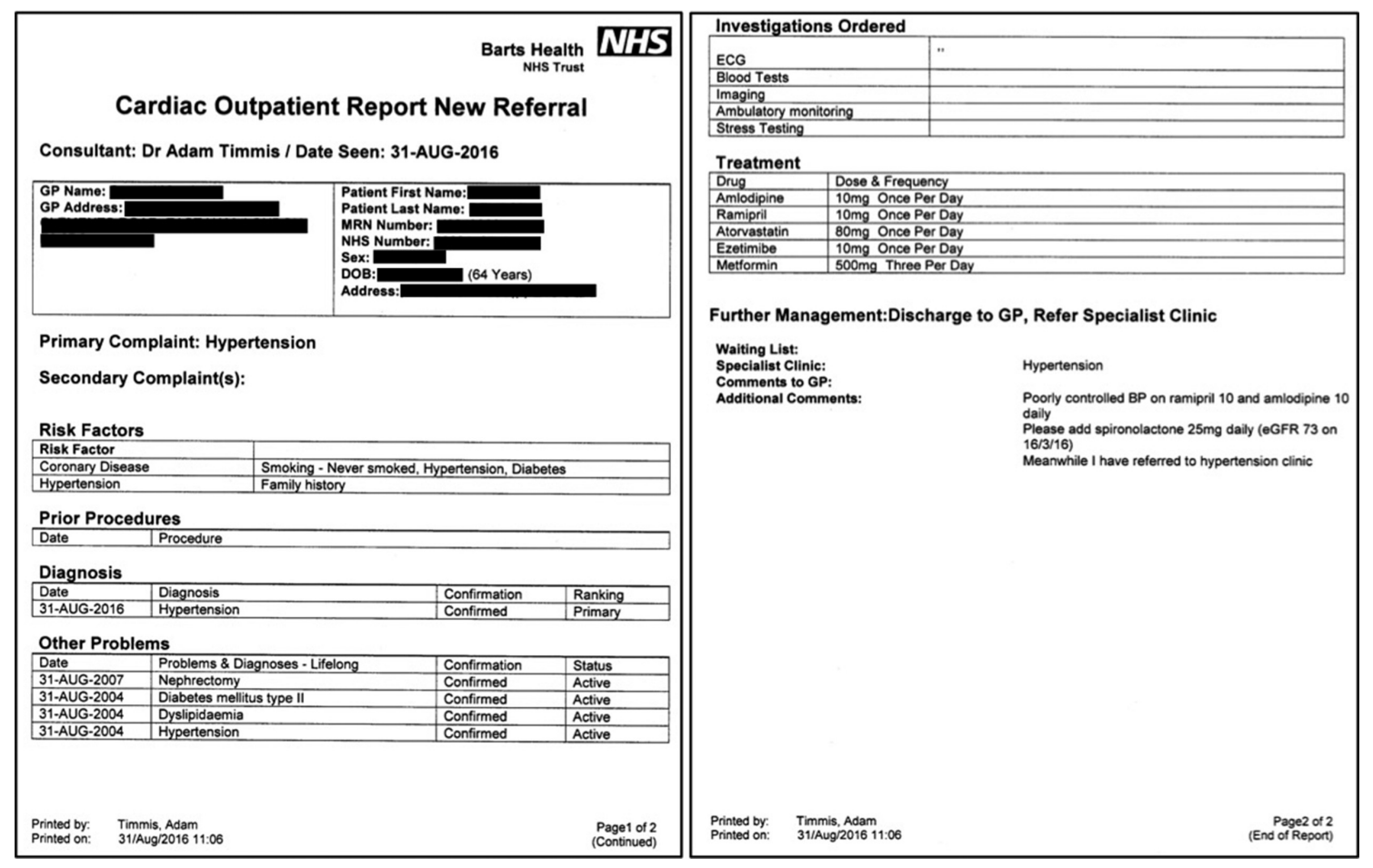

